\title{
Timescale of mass accretion in pre-main-sequence stars
}

\author{
D. Fedele ${ }^{1,2}$, M. E. van den Ancker $^{3}$, Th. Henning ${ }^{1}$, R. Jayawardhana ${ }^{4}$, and J. M. Oliveira ${ }^{5, \star}$ \\ 1 Max Planck Institut für Astronomie, Königstuhl 17, 69117 Heidelberg, Germany \\ e-mail: dfedele@mpia.de \\ 2 Departamento de Fisica Teórica, Facultad de Ciencias, Universidad Autónoma de Madrid, Cantoblanco, 28049 Madrid, Spain \\ 3 European Southern Observatory, Karl Schwarzschild Strasse 2, 85748 Garching bei München, Germany \\ 4 Department of Astronomy and Astrophysics, University of Toronto, 50 St. George Street, Toronto, ON, M5S 3H4, Canada \\ 5 Lennard-Jones Laboratories, School of Physical \& Geographical Sciences, Keele University, Staffordshire ST5 5BG, UK
}

Received 1 July 2009 / Accepted 17 November 2009

\section{ABSTRACT}

\begin{abstract}
We present the initial result of a large spectroscopic survey aimed at measuring the timescale of mass accretion in young, pre-mainsequence stars in the spectral type range K0-M5. Using multi-object spectroscopy with VIMOS at the VLT we identified the fraction of accreting stars in a number of young stellar clusters and associations of the ages of between 1-30 Myr. The fraction of accreting stars decreases from $\sim 60 \%$ at $1.5-2 \mathrm{Myr}$ to $\sim 2 \%$ at $10 \mathrm{Myr}$. No accreting stars are found after $10 \mathrm{Myr}$ at a sensitivity limit of $10^{-11} M_{\odot} \mathrm{yr}^{-1}$. We compared the fraction of stars showing ongoing accretion $\left(f_{\text {acc }}\right)$ to the fraction of stars with near-to-mid infrared excess $\left(f_{\text {IRAC }}\right)$. In most cases we find $f_{\text {acc }}<f_{\text {IRAC }}$, i.e., mass accretion appears to cease (or drop below detectable level) earlier than the dust is dissipated in the inner disk. At $5 \mathrm{Myr}, 95 \%$ of the stellar population has stopped accreting material at a rate of $\gtrsim 10^{-11} M_{\odot} \mathrm{yr}^{-1}$, while $\sim 20 \%$ of the stars show near-infrared excess emission. Assuming an exponential decay, we measure a mass accretion timescale $\left(\tau_{\text {acc }}\right)$ of $2.3 \mathrm{Myr}$, compared to a near-to-mid infrared excess timescale $\left(\tau_{\text {IRAC }}\right)$ of $3 \mathrm{Myr}$. Planet formation and/or migration, in the inner disk might be a viable mechanism to halt further accretion onto the central star on such a short timescale.
\end{abstract}

Key words. accretion, accretion disks - stars: pre-main sequence - planetary systems: protoplanetary disks

\section{Introduction}

Circumstellar disks around young, pre-main-sequence stars are the natural loci of planet formation (e.g. Henning 2008). Such a protoplanetary disk is formed during the collapse of the molecular cloud to conserve the initial angular momentum. It consists of interstellar gas and dust. The conventional planet formation model is the so-called core-accretion model (e.g. Pollack et al. 1996; Mordasini et al. 2008). In the simulations of Pollack et al. (1996), the formation of solar-system like planets takes up to 16 Myr. Giant planet formation is much faster ( $3 \mathrm{Myr})$ if planet migration is included (Alibert et al. 2004, 2005). Gravitational instability in the disk was also proposed as a viable scenario to form planets, especially in the outer disk (Boss 1997, 1998; Durisen 2007). If gravitational instabilities occur, the disk may fragment and form dense self-gravitating clumps which are the precursor of gas giant planets. In this model planet formation is very fast $(\lesssim 1 \mathrm{Myr})$.

Planet formation models face the fast evolution of protoplanetary disks. Star forming regions show a steady decline with time in the fraction of stars having infrared excess emission (e.g. Haisch et al. 2001, 2005; Bouwman et al. 2006; Hillenbrand 2008). This is thought to be caused by gradual clearing of dust in the inner disk. The growing consensus is that the warm small dust grains disappear within the first $5 \div 9 \mathrm{Myr}\left(\tau_{\text {dust }}\right)$. The quantity $\tau_{\text {dust }}$ is sometimes adopted as the disk lifetime. Although dust is essential to form a planet in terms of disk mass and hence dynamics and evolution, it accounts only for a small percentage of the disk mass. The bulk of the disk mass is thought to

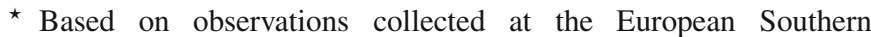
Observatory, Paranal, Chile (Proposal ID: 078.C-0282; 081.C-0208). be gaseous. At present, it is unclear whether the gas dissipation timescale $\left(\tau_{\text {gas }}\right)$ is similar to $\tau_{\text {dust }}$.

In this paper we report on a study aimed to determine the timescale for mass accretion $\left(\tau_{\text {acc }}\right)$ in protoplanetary disks. The timescale $\tau_{\text {acc }}$, i.e. the time at which the disk accretion phase ceases, provides a strong constraint on $\tau_{\text {gas }}$. Gas may still be present after $\tau_{\text {acc }}$, but the amount of the remaining gas and hence of the disk mass may be too low to be able to form giant planet(s). Our observational strategy, observations and data reduction are presented in Sect. 2. Analysis and results are presented in Sects. 3 and 4 respectively. In Sect. 5 we compare our result with literature data. Conclusions are drawn in Sect. 6.

\section{Method}

In order to measure $\tau_{\text {acc }}$ in a reliable way, we performed an optical spectroscopic survey of a large number of stars towards seven young stellar clusters (Table 1). Multi-object spectroscopy was performed with VIMOS $^{1}$ (LeFevre et al. 2003) at the VLT. No a priori target selection was made, instead we optimized the position of the slits in the multi-object masks in order to get the spectrum of as many stars as possible in the proximity of the cluster center. We did not aim at taking the spectrum of each (known) cluster member, but rather at building an unbiased inventory of the stellar population in a sub-region of the cluster down to a limiting magnitude of $V=21 \mathrm{mag}$. For each cluster we estimated the fraction of stars with ongoing mass accretion

\footnotetext{
1 http://www .eso.org/sci/facilities/paranal/ instruments/vimos/
} 
Table 1. Regions observed with VIMOS.

\begin{tabular}{lllllll}
\hline \hline \multirow{2}{*}{ Cluster } & Observing period & $\begin{array}{l}\text { RA(J2000) } \\
{[\mathrm{hh:mm:ss]}]}\end{array}$ & $\begin{array}{l}\text { Dec(J2000) } \\
{[\mathrm{dd}: \mathrm{mm}: \mathrm{ss}]}\end{array}$ & $\begin{array}{l}\text { Distance } \\
{[\mathrm{pc}]}\end{array}$ & $\begin{array}{l}A_{\mathrm{V}} \\
{[\mathrm{mag}]}\end{array}$ & $\begin{array}{l}\text { Sources } \\
{[N]}\end{array}$ \\
\hline$\sigma$ Orionis & Oct. 2006-Mar. 2007 & $05: 38: 00$ & $-02: 37: 00$ & 360 & 0.15 & 216 \\
NGC 6231 & Apr. 2008-Sep. 2008 & $16: 54: 10$ & $-41: 49: 30$ & 1240 & 1.36 & 573 \\
NGC 6531 & Apr. 2008-Sep. 2008 & $18: 04: 13$ & $-22: 29: 24$ & 1210 & 0.87 & 627 \\
ASCC 58 & Oct. 2006-Mar. 2007 & $10: 15: 07$ & $-54: 58: 12$ & 600 & 0.28 & 370 \\
NGC 2353 & Oct. 2006-Mar. 2007 & $07: 14: 30$ & $-10: 16: 00$ & 1120 & 0.22 & 316 \\
Collinder 65 & Oct. 2006-Mar. 2007 & $05: 26: 05$ & $+15: 41: 59$ & 310 & 0.40 & 198 \\
NGC 6664 & Apr. 2008-Sep. 2008 & $18: 36: 42$ & $-08: 13: 00$ & 1440 & 2.20 & 508 \\
\hline
\end{tabular}

Notes. The surveyed area for each cluster is $16^{\prime} \times 18^{\prime}$ centered at the coordinates listed above. The limiting magnitude is $V=21$ mag. MOS observations were carried out with the "HR-Orange" grism (maximum spectral coverage: $5200-7600 \AA$; $\lambda / \Delta \lambda=2150$ ) and a slit width of $1^{\prime \prime}$.

by analyzing the $\mathrm{H} \alpha$ line profile. Finally, the fraction of accreting stars was plotted against the age of the clusters.

In this paper we present the analysis of the mass accretion evolution. Results on individual clusters will be presented in a forthcoming paper.

The following criteria were adopted for the sample selection:

Age: the clusters have ages covering the interval of 2-30 Myr;

Distance and extinction: nearby and optically visible systems were selected to ensure the detection of low mass stars down to the spectral type M3 (at least);

Stellar density profile: to avoid any bias introduced by e.g.mass segregation, we only selected clusters known to have a spherical density profile;

Angular radius: (also linked to the previous point) only clusters with an angular size comparable to the field of view (FOV) of VIMOS were considered in order to minimize the number of contaminants.

For each cluster an area of $16^{\prime} \times 18^{\prime}$ was investigated acquiring spectra of $\sim 200-600$ representative objects in the central region of the cluster. Multiple dithered exposures were taken. Details about observations are given in Table 1 .

Data reduction was performed with IRAF and specific IDL routines. The spectra were reduced using a long-slit approach, i.e., extracting each spectrum separately. Due to distortion of the instrument night-time wavelength calibration exposures were taken. No night-time flat-field images were taken. For this reason we preferred to leave the MOS spectra uncorrected for flat field so no further source of noise was introduced. The following scheme was applied: bias subtraction, wavelength calibration, background removal and spectrum extraction using the Horne optimal extraction method (Horne 1986). Multiple dithered spectra were combined afterwards using a specific IDL routine: before combining the spectra, a scaling factor was applied to take into account slit losses in different exposures.

Figure 1 shows some examples of VIMOS spectra of our program stars.

\section{Analysis}

\subsection{Spectral type}

The spectral type of the objects classified as cluster members was derived. For this purpose we used the TiO index defined as

$\mathrm{TiO}(7140 \AA)=\frac{\mathrm{C}(7020-7050 \AA)}{\mathrm{TiO}(7125-7155 \AA)}$,

where $\mathrm{C}(7020-7050 \AA)$ is the spectral continuum computed between $7020-7050 \AA$, and $\mathrm{TiO}(7125-7155 \AA)$ is the intensity of the TiO molecular band absorption between 7125-7155 (Allen \& Strom 1995; Briceño et al. 1998; Oliveira et al. 2003). The TiO molecule is very sensitive to the stellar gravity and correlates very well with the spectral type in the range of K5-M6. We computed the spectral type of the VIMOS spectra, following the scheme of Jeffries et al. (2007). For earlier spectral types (K0-K5) we directly compared the VIMOS spectra with a set of standard star spectra which covered the same spectral range and had similar spectral resolution. The uncertainty on spectral type derived with these techniques is one sub-class.

In the following analysis we consider only stars with a spectral type between $\mathrm{K} 0$ and M5.

\subsection{Membership}

The cluster membership was established on the basis of the presence of two spectral features: emission of the $\mathrm{H} \alpha$ line at $6563 \AA$ and absorption of the Li $6708 \AA$ line. H $\alpha$ emission in young stars is either produced by mass accretion or by chromospheric activity. The presence of $\mathrm{Li}$ is also widely used as a youth indicator (e.g. Basri et al. 1991); Li is depleted very fast once the thermonuclear reactions begin in the core of the star. The abundance of $\mathrm{Li}$ in the stellar atmosphere is inversely proportional to the age of the star (Jeffries et al. 2007; Mentuch et al. 2008). This youth indicator is best suited for K- and M-type stars younger than 30 Myr (Jeffries et al. 2007). Some stars have strong Li absorption in their spectrum, but no $\mathrm{H} \alpha$ emission. These might be cluster members with no or a reduced chromospheric activity. We measured the $E W$ of $\mathrm{Li} 6708 \AA$ of these sources and compared this with the typical Li $6708 \AA E W$ measured for the certain members of the cluster $(0.2 \AA<E W[\mathrm{Li}]<0.6 \AA)$. We note that the lower limit of $E W$ Li measured here $(0.2 \AA)$ is an intermediate value assumed as a Li-indicator applied by other authors (e.g. Jeffries et al. 2007, $E W>0.3 \AA$; Winston et al. $2009, E W>0.1 \AA)$. In most of the cases the spectra show either a tiny $(E W \lesssim 0.1 \AA) \mathrm{H} \alpha$ emission or small absorption. In the latter case, the absorption is much smaller than the typical photospheric absorption for the same spectral type. The "reduced" $\mathrm{H} \alpha$ absorption might result from line veiling caused by the chromospheric activity. These stars are classified as cluster members.

\subsection{Signature of mass accretion}

To distinguish between chromospheric and accretion origin of the $\mathrm{H} \alpha$ emission, White \& Basri (2003) suggest a different threshold in $E W$ for different spectral types. Barrado y Navascués \& Martín (2003) have defined an empirical criterion based on the saturation limit of the chromospheric activity. 
D. Fedele et al.: Accretion Timescale in PMS stars

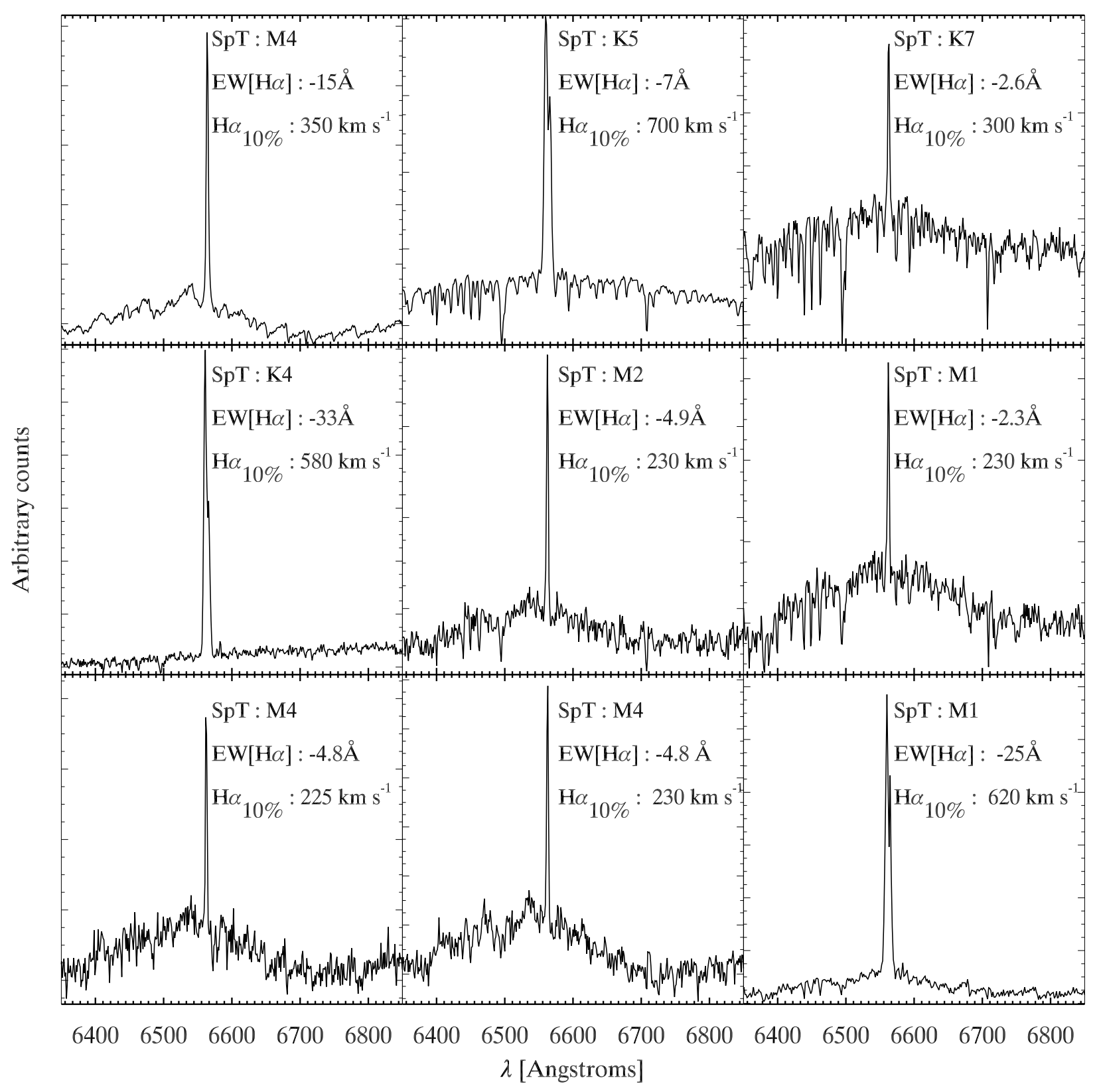

Fig. 1. Some examples of VIMOS spectra.

Another tool of investigation is the width of $\mathrm{H} \alpha$ at $10 \%\left(\mathrm{H} \alpha_{10 \%}\right)$ of the line peak (White \& Basri 2003; Natta et al. 2004; Jayawardhana et al. 2006; Flaherty \& Muzerolle 2008). As shown in Fig. 7 of White \& Basri (2003), accreting objects have a systematically larger value of $\mathrm{H} \alpha_{10 \%}$ compared to nonaccreting objects. Stars with $\mathrm{H} \alpha_{10 \%}>270 \mathrm{~km} \mathrm{~s}^{-1}$ are accreting material from a circumstellar disk. This criterion is valid down to a mass accretion rate of $10^{-10} \div 10^{-12} M_{\odot} \mathrm{yr}^{-1}$ (Natta et al. 2004). The actual limit on a detectable accretion rate depends on the stellar mass and age. As a conservative value we adopt $10^{-11} M_{\odot} \mathrm{yr}^{-1}$. In this work we used both the $E W[\mathrm{H} \alpha]$ and $\mathrm{H} \alpha_{10 \%}$ criterion to distinguish between accretion and chromospheric activity. This method is dependable to find a signature of accretion whenever $E W[\mathrm{H} \alpha]$ and $\mathrm{H} \alpha_{10 \%}$ are above or close to the thresholds (Jayawardhana et al. 2006). However, stars with values lower than the cutoff values may still be accreting at a low rate $\left(<10^{-11} M_{\odot} \mathrm{yr}^{-1}\right)$ and cannot be distinguished from pure chromospheric activity.
The spectral resolution of VIMOS is enough to measure the widening of the $\mathrm{H} \alpha$ line due to accetion. At $6563 \AA$ the minimum velocity resolvable with VIMOS is $140 \mathrm{~km} \mathrm{~s}^{-1}$. We measured the $E W$ and the $10 \%$ width of the $\mathrm{H} \alpha$ emission. The spectral continuum was computed using two spectral windows at both sides of the $\mathrm{H} \alpha$ line. The continuum level in correspondence to the emission line was interpolated using a first order polynomial. The spectra were then continuum-normalized and subtracted. $\mathrm{H} \alpha_{10 \%}$ was measured as the width of the $\mathrm{H} \alpha$ line at the height corresponding to $10 \%$ of the line peak. In the case of a narrow emission line and a low signal-to-noise spectrum, the $10 \%$ level might be confused with the neighbor-continuum fluctuations. To avoid this, the result was visually inspected. The major source of uncertainty for the measurements of $E W[\mathrm{H} \alpha]$ and $\mathrm{H} \alpha_{10 \%}$ is the determination of the underlying spectral continuum. The errors in $\mathrm{H} \alpha E W$ and $\mathrm{H} \alpha_{10 \%}$ were computed by taking the continuum error into account. For most of the spectra the signal-to-noise ratio close to the $\mathrm{H} \alpha$ line is $>10-20$. This translates into a precision 


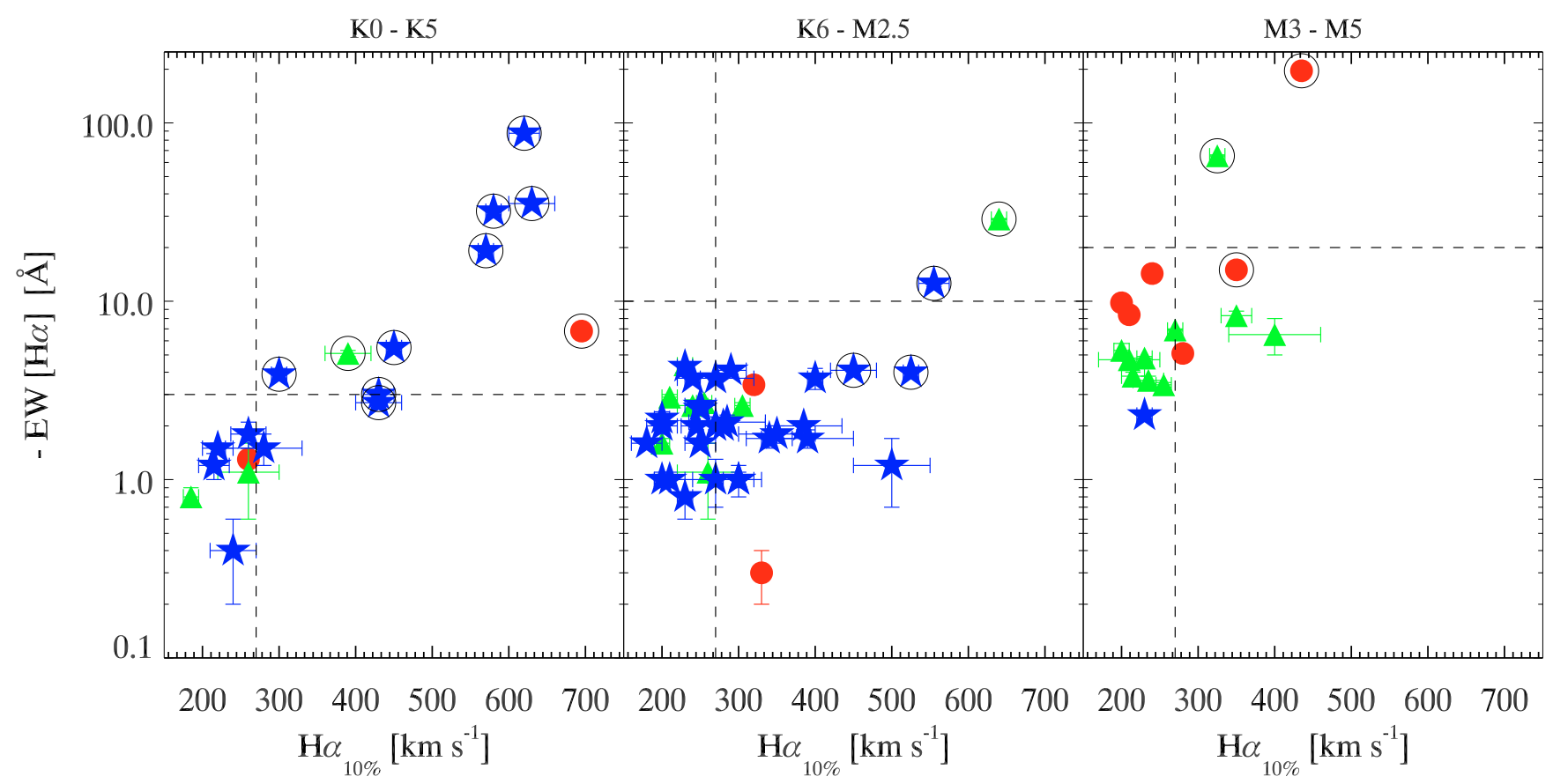

Fig. 2. $E W[\mathrm{H} \alpha]$ vs. $\mathrm{H} \alpha_{10 \%}$ for three different spectral type ranges. Vertical and horizontal dashed lines represent the thresholds for accreting stars. The different symbols refer to different clusters: dots - Sigma Ori, triangles - NGC 6531, stars - NGC 6231. Objects classified as accreting stars are over-plotted with open circles.

of $\sim 0.1 \AA$ and $5 \mathrm{~km} \mathrm{~s}^{-1}$ for the equivalent width and $\mathrm{H} \alpha \alpha_{10 \%}$, respectively. A further contribution of uncertainty is given by the instrument's spectral precision. We determined this by computing the full width half maximum and standard deviation for a number of arc lines in the proximity of the $\mathrm{H} \alpha$ line. The standard deviation is on the order of 5-7 $\mathrm{km} \mathrm{s}^{-1}$. We therefore assume $0.2 \AA$ and $10 \mathrm{~km} \mathrm{~s}^{-1}$ as a (conservative) lower limit to the uncertainty of $E W[\mathrm{H} \alpha]$ and $\mathrm{H} \alpha 10 \%$, respectively.

\section{Result}

None of the stars in regions older than $10 \mathrm{Myr}$ in our survey (ASCC 58, Collinder 65, NGC 2353 and NGC 6664) shows evidence of ongoing accretion (Table 2). Results for the youngest $(<10 \mathrm{Myr})$ regions are described below. In Fig. 2 we plot $E W$ $[\mathrm{H} \alpha]$ versus $\mathrm{H} \alpha_{10 \%}$ for three different spectral ranges. Vertical and horizontal dashed lines represent the thresholds for accreting stars. The different symbols refer to different clusters: dots $\sigma$ Ori, triangles - NGC 6531, stars - NGC 6231.

As shown in Fig. 2 there are a number of sources with $E W[\mathrm{H} \alpha]$ below the accretion threshold but with $\mathrm{H} \alpha_{10 \%}>$ $270 \mathrm{~km} \mathrm{~s}^{-1}$. If the line broadening is caused by mass accretion at a rate $>10^{-11} M_{\odot} \mathrm{yr}^{-1}$, we would expect to measure a large $E W$ as well. Besides accretion, stellar binarity and fast rotation might also be responsible for the line broadening. In order to decipher the nature of these sources we also investigated the presence of other emission lines which are associated with mass accretion (e.g. He I $5876 \AA$ A $6678 \AA$ \&; e.g. Beristain et al. 2001; Herczeg \& Hillenbrand 2008). In the presence of these accretion diagnostics, we can safely state that the star is accreting.

\section{$\sigma$ Ori}

Ten out of the 216 sources with spectra taken with VIMOS are classified as members of the $\sigma$ Ori cluster. All of them show emission in the $\mathrm{H} \alpha$ line as well as presence of Li. Two out of ten objects are clearly accreting stars according to the method mentioned above. A further object ( $\sigma$ Ori \# 1: RA $=84.584154$, Dec $=-2.633792$, Sp. Type M5) has a broad H $\alpha_{10 \%}\left(350 \mathrm{~km} \mathrm{~s}^{-1}\right)$ but $\mathrm{H} \alpha E W$ value $(15 \AA)$ below the accreting threshold. The presence of He emission lines $(5876 \AA E W=-0.75,6678 \AA$ $E W=-0.2)$ suggests the presence of hot gas in the vicinity of the star and hence is a signature of ongoing accretion (Beristain et al. 2001). The fraction of accreting objects in $\sigma$ Ori derived here is $3 / 10$ or $30 \% \pm 17 \% \%^{2}$. This agrees with the previous result of Zapatero Osorio et al. (2002, 30-40\%), Barrado y Navascués \& Martín (2003, $27 \pm 7 \%)$, Oliveira et al. (2006, 30\%). The good agreement with previous results validates our observational strategy despite the low number statistics.

\section{NGC 6231}

Out of 573 sources in the region of NGC 6231 we identified 78 objects as cluster members. The majority of these sources have a spectral type in the range of $\mathrm{K} 0-\mathrm{M} 5$ (there are three sources of an earlier spectral type) and show both $\mathrm{H} \alpha$ emission and strong $\mathrm{Li}$ absorption. Eleven out of 78 objects (all late K type) were identified based only on the presence of Li. Each of these spectra was compared with a standard star of an equal spectral type. All these spectra show either a tiny $(E W \sim 0.1 \AA) \mathrm{H} \alpha$ emission or small absoption. In the latter case, the absoption is much smaller than the typical photospheric absorption for the same spectral type. Three other objects have no $\mathrm{H} \alpha$, and the presence of the Li 6707.8 absorption is not clear. Seven out of the 75 K0-M5 sources in NGC 6231 are consistent with ongoing mass accretion with both diagnostics. Two more sources have $E W[\mathrm{H} \alpha]$ close to the accretion threshold, but large $\left(>270 \mathrm{~km} \mathrm{~s}^{-1}\right) \mathrm{H} \alpha_{10 \%}$ (panel $\mathrm{K} 0-\mathrm{K} 5$ in Fig. 2). Many stars with a spectral type in the range of K6-M2.5 have large $\mathrm{H} \alpha_{10 \%}$ but small $E W[\mathrm{H} \alpha]$. We inspected all of them and found two objects with a spectral type K7 showing

\footnotetext{
${ }_{2 \text { Computed as } \sqrt{N_{*, \text { acc }}} / N_{*, \text { total }}}$.
} 
D. Fedele et al.: Accretion Timescale in PMS stars

Table 2. Adopted age, spectral type range, $f_{\text {acc }}$ and $f_{\text {IRAC }}$ (when available) in Figs. 3 and 4.

\begin{tabular}{|c|c|c|c|c|c|c|c|}
\hline Cluster & $\begin{array}{l}\text { Age } \\
{[\mathrm{Myr}]}\end{array}$ & Sp.T range & $\begin{array}{l}f_{\text {acc }} \\
{[\%]}\end{array}$ & $\begin{array}{l}f_{\mathrm{IRAC}} \\
{[\%]}\end{array}$ & Age ref. & $f_{\text {acc }}$ ref. & $f_{\text {IRAC }}$ ref. \\
\hline rho Oph & 1 & K0-M4 & $50 \pm 16$ & & M05 & M05 & \\
\hline Taurus & 1.5 & K0-M4 & $59 \pm 9$ & 62 & M05 & M05 & $\mathrm{Ha} 05$ \\
\hline NGC 2068/71 & 2 & K1-M5 & $61 \pm 9$ & 70 & FM08 & FM08 & FM08 \\
\hline Cha I & 2 & K0-M4 & $44 \pm 8$ & $52-64$ & Lu08 & M05 & $\mathrm{Lu08}$ \\
\hline IC348 & 2.5 & K0-M4 & $33 \pm 6$ & 47 & L06 & M05 & L06 \\
\hline NGC 6231 & 3 & K0-M3 & $15 \pm 5$ & & S07 & this work & \\
\hline$\sigma$ Ori & 3 & K4-M5 & $30 \pm 17$ & 35 & $\mathrm{C} 08$ & this work & $\mathrm{He} 07$ \\
\hline Upper Sco & 5 & K0-M4 & $7 \pm 2$ & 19 & $\mathrm{C} 06$ & M05 & $\mathrm{C} 06$ \\
\hline NGC 2362 & 5 & K1-M4 & $5 \pm 5$ & 19 & D07 & D07 & D07 \\
\hline NGC 6531 & 7.5 & K4-M4 & $8 \pm 5$ & & P01 & this work & \\
\hline$\eta$ Cha & 8 & K4-M4 & $27 \pm 19$ & 50 & S09 & JA06 & S09 \\
\hline TWA & 8 & K3-M5 & $6 \pm 6$ & & D06 & JA06 & \\
\hline NGC 2169 & 9 & K5-M6 & $0^{+3}$ & & JE07 & JE07 & \\
\hline 25 Ori & 10 & K2-M5 & $6 \pm 2$ & & B07 & B07 & \\
\hline NGC 7160 & 10 & K0-M1 & $2 \pm 2$ & 4 & SA06 & SA05 & SA06 \\
\hline ASCC 58 & 10 & K0-M5 & $0^{+5}$ & & K05 & this work & \\
\hline$\beta \mathrm{Pic}$ & 12 & K6-M4 & $0^{+13}$ & & ZS04 & JA06 & \\
\hline NGC 2353 & 12 & K0-M4 & $0^{+6}$ & & K05 & this work & \\
\hline Collinder 65 & 25 & K0-M5 & $0^{+7}$ & & K05 & this work & \\
\hline Tuc-Hor & 27 & K1-M3 & $0^{+8}$ & & ZSO4 & JA06 & \\
\hline NGC 6664 & 46 & K0-M1 & $0^{+4}$ & & S82 & this work & \\
\hline
\end{tabular}

References. Schmidt (1982, S82), Park et al. (2001, P01), Hartmann et al. (2005, Ha05), Kharchenko et al. (2005, K05), Mohanty et al. (2005, M05), Sicilia-Aguilar et al. (2005, SA05), Carpenter et al. (2006, C06), Lada et al. (2006, L06), Jayawardhana et al. (2006, JA06), Sicilia-Aguilar et al. (2006, SA06), Dahm \& Hillenbrand (2007, D07), Briceño et al. (2007, B07), Jeffries et al. (2007, JE07), Hernández et al. (2007, He07), Sana et al. (2007, S07), Caballero (2008, C08), Flaherty \& Muzerolle (2008, FM08), Luhman et al. (2008, L08), Sicilia-Aguilar et al. (2009, S09), Zuckerman \& Song (2004, ZS04).

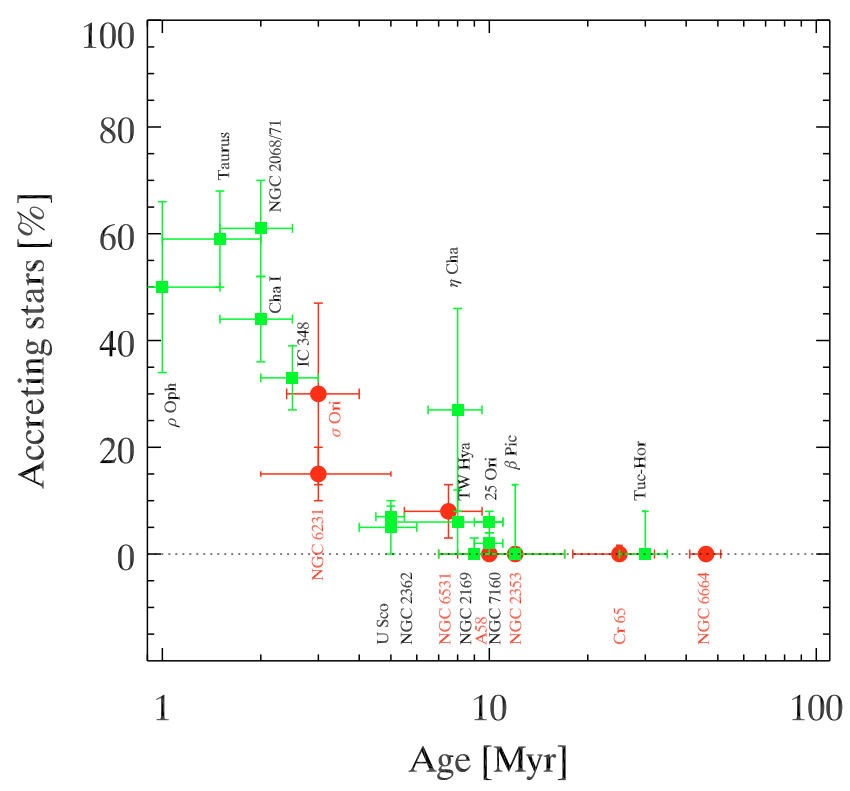

Fig. 3. Accreting stars-frequency as a function of age. New data (based on the VIMOS survey) are shown as (red) dots, literature data as (green) squares. Colored version is available in the electronic form.

He I $5876 \AA$ in emission $(E W=-0.5 \AA,-0.6 \AA$ respectively). The evidence of large $\mathrm{H} \alpha_{10}$ \% together with the He I emission is most likely due to ongoing mass accretion, and these two stars are classified as accreting stars. We estimate a fraction of accreting stars in NGC 6231 of $11 / 75$ or $15( \pm 5 \%)$. We warn the reader that this might be a lower limit to the actual fraction of accreting stars; further investigation is needed to disentagle the nature (accretion vs binarity/rapid rotation) of the systems with large $\mathrm{H} \alpha_{10 \%}\left(>300 \mathrm{~km} \mathrm{~s}^{-1}\right)$ but low $E W[\mathrm{H} \alpha]$.

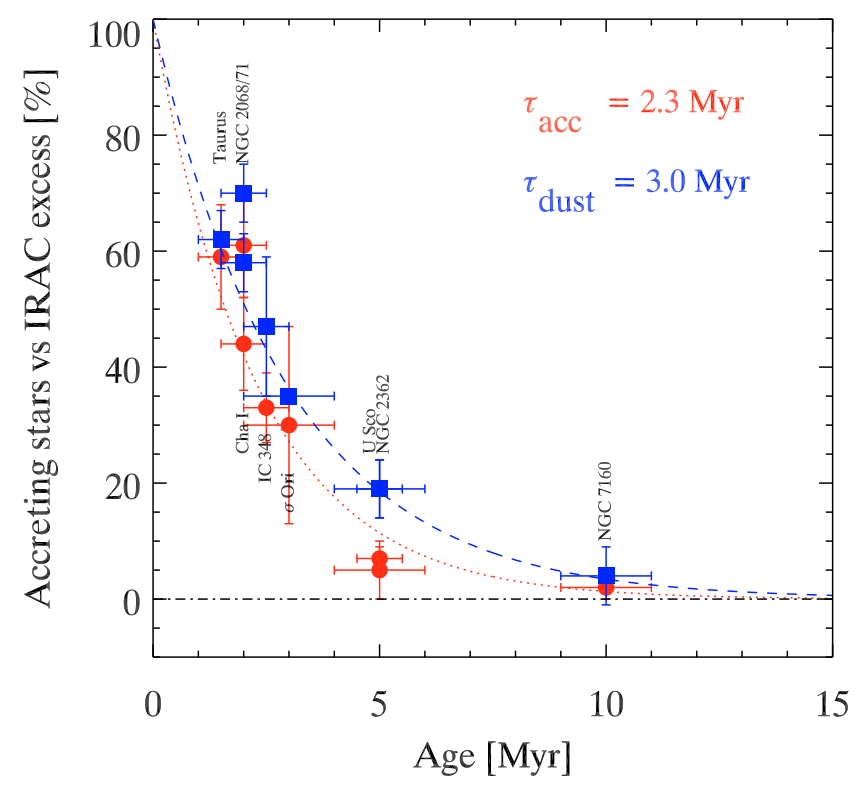

Fig. 4. $f_{\text {acc }}$ (dots) versus $f_{\text {IRAC }}$ (squares) and exponential fit for $f_{\text {acc }}$ (dotted line) and for $f_{\mathrm{IRAC}}$ (dashed line).

\section{NGC 6531}

We identified 26 cluster members in NGC 6531 based on the presence of $\mathrm{H} \alpha$ emission and presence of $\mathrm{Li} .13$ other sources show presence of $\mathrm{Li} 6708 \AA$, but have $\mathrm{H} \alpha$ in absorption. As in the case of NGC 6231, these might be cluster members with no or a reduced chromospheric activity. We measured the $E W$ of $\mathrm{Li}$ $6708 \AA$ of these 13 sources and compared them with the typical $E W$ of the 26 stars in NGC 6531 showing also $\mathrm{H} \alpha$ emission 
$(0.2 \AA<E W \mathrm{Li}<0.6 \AA)$. Ten of the 13 sources have similar $E W$ and are likely cluster members. The remaining three sources have $E W$ between $0.1-0.2 \AA$. Moreover, these are characterized by strong absorption lines at 5778, 5796, 6284, $6614 \AA$, which are produced by diffuse interstellar absorption bands (DIBs). These are probably located in the background of the cluster and are classified as non-members. Out of the 36 identified members, three objects show broad and strong $\mathrm{H} \alpha$ emission consistent with mass accretion. One further object is classified as an uncertain accreting star. The fraction of accreting stars is then $8 \pm 5 \%$. Given the large $\mathrm{H} \alpha_{10 \%}\left(>300 \mathrm{~km} \mathrm{~s}^{-1}\right)$ of two objects in the spectral range of M3-M5, the fraction of accreting stars computed here might be a lower limit. Follow-up observations are needed for these two sources.

\section{Discussion}

The fraction of accreting stars $\left(f_{\text {acc }}\right)$ for each cluster is listed in Table 2 and plotted in Fig. 3 together with literature data.

The aim of this paper is to trace the evolution of mass accretion with time. A critical point is the age of the clusters. We adopted the most recent age measurements (Table 2). For NGC 6231 Sana et al. (2007) find that the bulk of the CTTS has an age between 2-4 Myr, although with a large age spread. The most recent study of $\sigma$ Ori suggests an age of 3 Myr (e.g. Caballero 2008). For NGC 6531 Park et al. (2001) measure an age of $7.5 \pm 2 \mathrm{Myr}$ in agreement with Forbes (1996), who measure $8 \pm 3$ Myr. For the remaining clusters we adopt the age provided by WEBDA ${ }^{3}$. For these clusters the age is estimated by fitting the zero-age-main-sequence (ZAMS) to the brightest stars of the cluster. We take an accuracy of $30 \%$ as a conservative assumption.

In Fig. 3 the fraction of accreting stars as function of cluster age is shown. The results obtained with VIMOS are shown as filled circles. Measurements of $f_{\text {acc }}$ exist in the literature for some clusters. These are are shown as filled squares in Fig. 3 and are listed in Table 2. We considered only determinations of $f_{\text {acc }}$, using the method of White \& Basri (2003) and/or Barrado y Navascués \& Martín (2003). The fraction of accreting stars may vary with spectral type. In the case of Upper Sco Mohanty et al. (2005) e.g., found an increase of $7 \%( \pm 2 \%)$ for a spectral type earlier than M4 to $20 \%( \pm 10 \%)$ for later spectral types. For the youngest regions, $\rho$ Oph, Taurus, Cha I and IC 348, Mohanty et al. (2005) find no appreciable variation, within errors, in the fraction of accreting sources between the two mass ranges. For consistency with our survey we include here only the results for the spectral types between K0-M5.

\subsection{Evolution of $f_{\mathrm{acc}}$}

The fraction of accreting stars decreases quickly with time within the first $10 \mathrm{Myr}$. There is a clear trend from the 1.5-2 Myr old regions $\left(f_{\text {acc }}=60 \%\right.$ ) down to the $10 \mathrm{Myr}$ old clusters (mean $f_{\text {acc }}=2 \%$ ). At the age of 5 Myr the mean $f_{\text {acc }}$ is with $\sim 5 \%$ drastically lower than the 3 Myr old regions of $\sigma$ Ori and NGC 6231 (average 24\%). No accreting stars are found beyond $10 \mathrm{Myr}$. This agrees well with previous measurements of the mass accretion timescale by e.g. Mohanty et al. (2005) and Jayawardhana et al. (2006). There are some outliers. The $\eta$ Cha cluster shows higher frequency of accretors compared to clusters of an equal

\footnotetext{
${ }^{3}$ http://www. univie.ac.at/webda/, operated at the Institute for Astronomy of the University of Vienna.
}

age (e.g. Sicilia-Aguilar et al. 2009). Through this particular association a similar behavior in the disk frequency is made apparent (from near-infrared excess). Age and distance are unlikely to be wrong for this well-known system. Moraux et al. (2007) suggest that most of the low-mass members of the association have been dispersed by dynamical evolution. The current list of members of $\eta$ Cha might be biased towards infrared-excess/ $\mathrm{H} \alpha$ emitting sources. Inversely the very young region of $\rho$ Oph lies below the trend. The statistics in this region are limited by extinction, which may obscure part of the stellar population (e.g. Mohanty et al. 2005). Moreover, the result of Mohanty et al. (2005) might be contaminated by an older population of stars; objects in the periphery of $\rho$ Oph core appear older than stars in the core itself (Wilking et al. 2005). In the following analysis we will not include $\rho$ Oph and $\eta$ Cha.

NGC 6231 (2-4 Myr) also appears to have a low $f_{\text {acc }}$ for its age. Interestingly, this region hosts many $\mathrm{O}$ and $\mathrm{B}$ stars. External photoevaporation (by means of the $\mathrm{O}, \mathrm{B}$ stars) may accelerate the disk dissipation.

\section{2. $f_{\mathrm{acc}} v s . f_{\mathrm{IRAC}}$}

In this section we compare the fraction of accreting stars to the fraction of stars with near-to-mid infrared excess $\left(f_{\text {IRAC }}\right)$. The latter is measured as the fraction of stars having infrared excess in the Spitzer/IRAC [3.6] - [8.0] bands over the total fraction of stars in the cluster. The definition of an excess source is based on the slope $(\alpha)$ of the infrared spectral energy distribution determined from the IRAC [3.6] - [8.0] bands (Lada et al. 2006)

$\alpha_{\text {IRAC }}=\frac{\mathrm{d}\left(\log \left(\lambda F_{\lambda}\right)\right)}{\mathrm{d}(\log (\lambda))} ; \quad 3.6 \mu \mathrm{m}<\lambda<8.0 \mu \mathrm{m}$.

Stars with $-1.8<\alpha<0$ are classified as class II (e.g. Hernández et al. 2007). In order to coherently compare $f_{\text {IRAC }}$ with $f_{\text {acc }}$ we refer only to stars of a spectral type between K0-M5. Whenever $f_{\text {IRAC }}$ was measured as a function of the stellar mass rather than the spectral type we only consider stars of a mass of between $0.1-1.2 M_{\odot}$. By applying these selection criteria we find the following results for $f_{\text {IRAC }}$ (Table 2): Taurus $-62 \%$ (Hartmann et al. 2005), NGC 2068/71 - 70\% (Flaherty \& Muzerolle 2008), Cha I $-52 \% \div 64 \%$ (Luhman et al. 2008), IC $348-47 \%$ (Lada et al. 2006), $\sigma$ Ori - 35\% (Hernández et al. 2007), Upper Sco 19\% (Carpenter et al. 2006), NGC 2362 - 19\% (Dahm \& Hillenbrand 2007), $\eta$ Cha - 50\% (Sicilia-Aguilar et al. 2009) and NGC 7160 - 4\% (Sicilia-Aguilar et al. 2006).

Interestingly enough, we find for some clusters a lower fractional value of stars with evidence of ongoing accretion compared to stars with near-to-mid infrared excess. For example among the 64 K0-M5 stars identified by Flaherty \& Muzerolle (2008) in NGC $2068 / 71,39(61 \%)$ of these show a clear signature of mass accretion, while $45(70 \%)$ stars have IRAC excess. We note that two of the non-accreting stars in their sample are identified by strong IRAC excess (ID 416, 843 in Flaherty \& Muzerolle 2008), while one transitional object (ID 281) is a strong accretor. Similarly, for $\sigma$ Ori we measure an $f_{\text {acc }}=$ $30 \%$ while $f_{\text {IRAC }}=35 \%$. This is consistent with the result of Damjanov et al. (2007) in Chameleon I. They found a small population of non-accreting objects bearing a dusty inner disk.

In Fig. 4 we plot $f_{\text {acc }}$ vs. $f_{\mathrm{IRAC}}$ for the regions where both quantities are known. Assuming an exponential decay we fit $f_{\text {acc }}$ and $f_{\mathrm{IRAC}}$ with the following function

$f_{i}=C \cdot \exp \left(-t / \tau_{i}\right)$ 
where $C$ is a constant and it is normalized assuming $f_{\text {acc }}=$ $f_{\text {IRAC }}=100 \%$ for $t=0$. The fit gives an accretion timescale of $\tau_{\text {acc }}=2.3$ Myr and a near-to-mid infrared excess timescale of $\tau_{\text {IRAC }}=3 \mathrm{Myr}$.

\subsection{Inner disk dissipation and planet formation}

Mass accretion and dust dispersion in the inner disk appear to have different timescales. At an age of $5 \mathrm{Myr}, 95 \%$ of the total stellar population have stopped accreting material at a rate $\gtrsim 10^{-11} M_{\odot} \mathrm{yr}^{-1}$, while $\sim 20 \%$ of the disks still retain enough dust to produce detectable infrared excess. In order to sustain the mass accretion at a rate larger than $10^{-11} M_{\odot} \mathrm{yr}^{-1}$, a gas disk reservoir is required. This implies that at an age of $\tau_{\text {acc }}=2.3 \mathrm{Myr}$ most of the disk mass is drastically reduced while at an age of 5 Myr only $5 \%$ of the disks have enough gas mass to keep on accreting material onto the central star. Models of viscous evolution (e.g. Hartmann et al. 1998) predict a steady decrease of the mass accretion rate with time, and this might be an explanation for the bimodal evolution of $f_{\text {acc }}$ and $f_{\text {IRAC. }}$. However, the timescale for viscous evolution to stop accretion (>10 Myr) is much longer than our estimate of $\tau_{\text {acc }}(2.3 \mathrm{Myr})$. The efficiency of viscous evolution might be enhanced if photoevaporation is also taken into account as first argued by Clarke et al. (2001). Their so-called "UV-switch" model is very efficient in stopping further accretion as the accretion rate drops below the photoevaporation rate. The advantage of this is the shorter accretion timescale. But this model also has a drawback, namely that disk dissipation following the end of the accretion phase should occur on a viscous timescale $\left(10^{5} \mathrm{yr}\right)$. This is in contrast with the relatively high number of stars still retaining their dusty disk (e.g. $20 \%$ at $5 \mathrm{Myr}$ ) after accretion ceases.

Another possible explanation is that the accretion onto the central star is stopped by planet formation and migration in the inner disk. If a giant planet forms/migrates to the inner region of the disk, it may stop further accretion onto the star. In this respect, recent self-consistent numerical simulations of planet formation by for instance Thommes et al. (2008) are consistent with this scenario. In these simulations planetesimal formation occurs over a large range of disk radii. As soon as the cores of giant planets form, they migrate to small radii $(<1 \mathrm{AU})$. This happens very fast, within 1-2 Myr. The disk surface density drops to zero, and so does the accretion onto the star, within the planet's orbit. Dust grains beyond the orbit of the planet might instead still absorb and re-emit the stellar light and produce the measured IRAC excess.

\section{Conclusion}

We presented an analysis of the evolution of the mass accretion in PMSs in the spectral type range K0-M5. These are the main results: (1) the fraction of stars with ongoing mass accretion decreases fast with time, going from $\sim 60 \%$ at $1.5-2 \mathrm{Myr}$ down to $\sim 2 \%$ at $10 \mathrm{Myr}$; (2) this fraction is systematically lower than the fraction of stars showing near-to-mid infrared excess; (3) mass accretion and dust dissipation in the inner disk appear to have different characteristic timescales of 2.3 and 3 Myr respectively; (4) within $5 \mathrm{Myr}$ the mass accretion rate of $95 \%$ of the stellar population drops below our detection limit of $10^{-11} M_{\odot} \mathrm{yr}^{-1}$. While viscous evolution and photoevaporation might be unable to slow down accretion (and leave a substantial dust mass) on such a short timescale, planet formation and/or migration in the inner disk (few $\mathrm{AU}$ ) might be a viable mechanism to halt further accretion onto the central star.

Acknowledgements. We thanks the ESO staff for performing the VIMOS observations in service mode. This research has made use of the SIMBAD database, operated at CDS, Strasbourg, France. We are grateful to the anonymous referee for his comments and suggestions.

\section{References}

Alibert, Y., Mordasini, C., \& Benz, W. 2004, A\&A, 417, L25

Alibert, Y., Mordasini, C., Benz, W., \& Winisdoerffer, C. 2005, A\&A, 434, 343

Allen, L. E., \& Strom, K. M. 1995, AJ, 109, 1379

Barrado y Navascués, D., \& Martín, E. L. 2003, AJ, 126, 2997

Basri, G., Martin, E. L., \& Bertout, C. 1991, A\&A, 252, 625

Beristain, G., Edwards, S., \& Kwan, J. 2001, ApJ, 551, 1037

Boss, A. P. 1997, Science, 276, 1836

Boss, A. P. 1998, ApJ, 503, 923

Bouwman, J., Lawson, W. A., Dominik, C., et al. 2006, ApJ, 653, L57

Briceño, C., Hartmann, L., Stauffer, J., \& Martín, E. 1998, AJ, 115, 2074

Briceño, C., Hartmann, L., Hernández, J., et al. 2007, ApJ, 661, 1119

Caballero, J. A. 2008, MNRAS, 383, 375

Carpenter, J. M., Mamajek, E. E., Hillenbrand, L. A., \& Meyer, M. R. 2006, ApJ, 651, L49

Clarke, C. J., Gendrin, A., \& Sotomayor, M. 2001, MNRAS, 328, 485

Dahm, S. E., \& Hillenbrand, L. A. 2007, AJ, 133, 2072

Damjanov, I., Jayawardhana, R., Scholz, A., et al. 2007, ApJ, 670, 1337

Durisen, R. H. E. A. 2007, in PPV, ed. B. Reipurth, D. Jewitt, \& K. Keil, 607

Flaherty, K. M., \& Muzerolle, J. 2008, AJ, 135, 966

Forbes, D. 1996, AJ, 112, 1073

Haisch, Jr., K. E., Lada, E. A., \& Lada, C. J. 2001, ApJ, 553, L153

Haisch, Jr., K. E., Jayawardhana, R., \& Alves, J. 2005, ApJ, 627, L57

Hartmann, L., Calvet, N., Gullbring, E., \& D’Alessio, P. 1998, ApJ, 495, 385

Hartmann, L., Megeath, S. T., Allen, L., et al. 2005, ApJ, 629, 881

Henning, T. 2008, Phys. Scr., T 130, 014019

Herczeg, G. J., \& Hillenbrand, L. A. 2008, ApJ, 681, 594

Hernández, J., Hartmann, L., Megeath, T., et al. 2007, ApJ, 662, 1067

Hillenbrand, L. A. 2008, Phys. Scr., T 130, 014024

Horne, K. 1986, PASP, 98, 609

Jayawardhana, R., Coffey, J., Scholz, A., Brandeker, A., \& van Kerkwijk, M. H. 2006, ApJ, 648, 1206

Jeffries, R. D., Oliveira, J. M., Naylor, T., Mayne, N. J., \& Littlefair, S. P. 2007, MNRAS, 376, 580

Kharchenko, N. V., Piskunov, A. E., Röser, S., Schilbach, E., \& Scholz, R.-D. 2005, A\&A, 438, 1163

Lada, C. J., Muench, A. A., Luhman, K. L., et al. 2006, AJ, 131, 1574

LeFevre, O., Saisse, M., Mancini, D., et al. 2003, in SPIE Conf. Ser. 4841, ed. M. Iye, \& A. F. M. Moorwood, 1670

Luhman, K. L., Allen, L. E., Allen, P. R., et al. 2008, ApJ, 675, 1375

Mentuch, E., Brandeker, A., van Kerkwijk, M. H., Jayawardhana, R., \& Hauschildt, P. H. 2008, ApJ, 689, 1127

Mohanty, S., Jayawardhana, R., \& Basri, G. 2005, ApJ, 626, 498

Moraux, E., Lawson, W. A., \& Clarke, C. 2007, A\&A, 473, 163

Mordasini, C., Alibert, Y., Benz, W., \& Naef, D. 2008, in ASPC Seri. 398, ed.

D. Fischer, F. A. Rasio, S. E. Thorsett, \& A. Wolszczan, 235

Natta, A., Testi, L., Muzerolle, J., et al. 2004, A\&A, 424, 603

Oliveira, J. M., Jeffries, R. D., Devey, C. R., et al. 2003, MNRAS, 342, 651

Oliveira, J. M., Jeffries, R. D., van Loon, J. T., \& Rushton, M. T. 2006, MNRAS, 369,272

Park, B.-G., Sung, H., \& Kang, Y. H. 2001, J. Korean Astron. Soc., 34, 149

Pollack, J. B., Hubickyj, O., Bodenheimer, P., et al. 1996, Icarus, 124, 62

Sana, H., Rauw, G., Sung, H., Gosset, E., \& Vreux, J.-M. 2007, MNRAS, 377, 945

Schmidt, E. G. 1982, AJ, 87, 1197

Sicilia-Aguilar, A., Hartmann, L. W., Hernández, J., Briceño, C., \& Calvet, N. 2005, AJ, 130, 188

Sicilia-Aguilar, A., Hartmann, L. W., Fürész, G., et al. 2006, AJ, 132, 2135

Sicilia-Aguilar, A., Bouwman, J., Juhász, A., et al. 2009, ApJ, 701, 1188

Thommes, E. W., Matsumura, S., \& Rasio, F. A. 2008, Science, 321, 814

White, R. J., \& Basri, G. 2003, ApJ, 582, 1109

Wilking, B. A., Meyer, M. R., Robinson, J. G., \& Greene, T. P. 2005, AJ, 130, 1733

Winston, E., Megeath, S. T., Wolk, S. J., et al. 2009, AJ, 137, 4777

Zapatero Osorio, M. R., Béjar, V. J. S., Pavlenko, Y., et al. 2002, A\&A, 384, 937

Zuckerman, B., \& Song, I. 2004, ARA\&A, 42, 685 